\title{
Comparative Analysis of C-repeat Binding Factors (CBFs) in Tomato and Arabidopsis
}

\author{
Parviz Heidari ${ }^{1}$ \\ https://orcid.org/0000-0003-4716-0143 \\ ${ }^{1}$ Shahrood University of Technology, Faculty of Agriculture, Department of Agronomy and Plant \\ Breeding; Shahrood, Iran
}

Received: 2018.12.07; Accepted: 2019.06.16.

* Correspondence: heidarip@shahroodut.ac.ir; Tel.: +98-9120734034

\section{HIGHLIGHTS}

- This study focused on the investigation CBF genes in Arabidopsis and tomato.

- The different cis-regulatory elements were observed in promoter sites.

- $\quad$ SICBF1 and AtCBF1 genes showed differential expression under cold stress.

- $\quad$ AtCBF1 is more induced than SICBF1 under cold stress.

Abstract: Cold stress is one of the limiting factors of plant production that plants use different mechanisms for cold tolerance. CBF genes play critical role to regulate the cold responsive genes. To better understand of CBF gene functions, the tomato-CBFs and Arabidopsis-CBFs were evaluated using bioinformatics tools, and finally the expression patterns of SICBF1 gene were analyzed under 10 and $4^{\circ} \mathrm{C}$ in two contrasting tomato species (Solanum lycopersicum and $S$. habrochaites). The different cis regulatory elements were observed in promoter region of SICBF1 and AtCBF1 genes, and ICE1, COR and HOS1 proteins exhibited high interaction with CBFs. The results of Real time PCR of SICBF1 exhibited that under 10 and $4{ }^{\circ} \mathrm{C}$, SICBF1 was down regulated in cold sensitive tomato genotype while it was slightly up-regulated in cold tolerant genotype at $4{ }^{\circ} \mathrm{C}$. The results showed that the SICBF1 and AtCBF1 genes have differential expression in cold stress.

Keywords: Cold stress; CBF genes; Real time PCR; Tomato; Bioinformatics analysis.

\section{INTRODUCTION}

Environmental stresses such as low temperature $\left(<18^{\circ} \mathrm{C}\right)$, high temperature, drought and high salinity have negative effects on plant growth, development and performance $[1,2]$. To adapt to unfavorable conditions such as cold stress, plants use the different mechanisms including molecular and physiological responses [3]. Transcription factors (TFs) as a molecular response have key role to regulate the expression patterns of target genes under 
Heidari, P.

biotic and abiotic stresses [4]. The AP2/ERF superfamily is a large TF family that is involved in the response to environmental stresses [5]. C-repeat binding factors (CBFs), also known as DREB, are members of AP2/ERF superfamily that play a fundamental role in regulation of cold-responsive genes and cold acclimation [6,7]. During cold stress in Arabidopsis, CBF1/DREB1B, CBF3/DREB1A, and CBF2/DREB1C are induced, and they bind the regulatory CRT/DRE element with conserved core sequence (5'-CCGAC-3') of promoter region in cold-regulated genes $[7,8]$. Previous studies illustrated that CBFs are induced under low temperature in barley [9], rice [10], Arabidopsis [11, 12] and wheat [13].

Tomato (Solanum lycopersicum) is one of the largest angiosperm genera and includes annual and perennial plants that most genotypes of tomato are sensitive to low temperature [14]. Three CBFs (SICBF1, 2 and 3) were identified in tomato that SICBF1 is induced in low temperature [15]. Recently, Li et al. generated slcbf1 mutant lines in tomato by the CRISPR/Cas9 system that the abscisic acid, methyl jasmonate, zeatin riboside and protein contents were decreased in slcbf1 mutants and wild type showed more cold resistance than mutant lines [16]. However, the overexpression of SICBF/in tomato could not increase the cold tolerance, but SICBF1 increased the cold stress tolerance in Arabidopsis transgenic plants [15]. It seems that the function of SICBF1 in tomato is different from other plant species such as Arabidopsis, and its role is still unknown under cold stress. To understand the role of SICBF1 in tomato during low temperature stresses, the expression patterns of SICBF1 in tomato genotypes (cold sensitive and cold tolerant genotypes) were analyzed at $10^{\circ} \mathrm{C}$ and $4{ }^{\circ} \mathrm{C}$ and also the Arabidopsis-CBFs and Tomato-CBFs were compared using bioinformatics tools. The results of this research provide the new information of SICBF1-regulatory mechanisms during the cold stresses.

\section{MATERIAL AND METHODS}

\section{Sequence analysis}

The protein sequences of CBF in tomato, Arabidopsis, wheat, barley, rice and potato were retrieved using NCBI database [17]. The some physicochemical characteristics of studied proteins such as molecular weight (MW) and isoelectric point (pl) were predicted using ProtParam tool [18], and the subcellular location of CBF proteins was predicted by Plant-mPLoc tool [19]. The STRING database [20] was used to predict the protein-protein interaction of tomato-CBFs and Arabidopsis-CBFs.

\section{Phylogenetic analysis}

The amino acid sequence of CBF proteins in tomato, Arabidopsis, potato and some important monocots such as wheat, barley and rice was used to construct the phylogenetic analysis using neighbor-joining method of MEGA7 software [21].

\section{Cis-acting regulatory elements analysis}

To identify the cis-regulatory elements in promoter regions, the $1000 \mathrm{bp}$ of upstream the start codon of SICBF1 and AtCBF1 were analyzed using Plant CARE [22].

\section{Microarray analysis}

The published data of genes expression profile of Arabidopsis were used to consider the expression patterns of AtCBF1-3 genes under abiotic stresses. The microarray data of AtCBF1-3 genes under cold, drought and salt stresses were obtained using the affymetrix Arabidopsis ATH1 genome array (10615 samples) from Genevestigator database [23]. 


\section{Plant materials and growth conditions}

Seeds of Solanum lycopersicum cv. Moneymaker (as cold sensitive) and $S$. habrochaites, LA1777 (as cold tolerant) were sown in 18 soil plates at $23 \pm 1^{\circ} \mathrm{C}$ under $14 \mathrm{~h}$ photoperiod duration. After six weeks, the 12 plates of tomato seedlings were transferred to two different growth chambers at set-point temperatures of $10 \pm 1^{\circ} \mathrm{C}$ and $4 \pm 1^{\circ} \mathrm{C}$ and 6 plates remained at $23 \pm 1^{\circ} \mathrm{C}$. After three days, the whole shoots of each plate were collected and stored in liquid nitrogen and transferred to $-80^{\circ} \mathrm{C}$.

\section{RNA extraction and cDNA preparation}

The leaves from three individual tomato seedlings were powdered in liquid nitrogen and then the total RNA was extracted by RNX TM -Plus (Sinaclon). The quantity and quality of extracted RNA were determined using a Nano Photometer (Implen N50). Reverse transcription was carried out using $1 \mu \mathrm{g}$ total RNA treated with RNase-free DNase I (Thermo Scientific) and reverse transcriptase (Roche, Germany) according to instructions of manufacture. The primers for SICBF1 (F: 5'- CCTGCTTCCTCCAACTCTAAA -3' and R: 5'CTCATCCACGAAGTCACTACTC $\left.-3^{\prime}\right)$ and EF-1- $\alpha$ (F: $5^{\prime}-$ GGAACTTGAGAAGGAGCCTAAG -3' and R: 5'- CAACACCAACAGCAACAGTCT -3') as reference gene were designed and evaluated using primer3 plus [24].

\section{Real time PCR}

The real time PCR was run with Applied Biosystems StepOne TM using RealQ Plus 2x Master Mix Green high ROX TM (Ampliqon) according to instructions of manufacture. The conditions of real time PCR were combined: $95^{\circ} \mathrm{C}$ for $10 \mathrm{~min}$, followed by 35 cycles at $95^{\circ} \mathrm{C}$ for $15 \mathrm{~s}$ and $61^{\circ} \mathrm{C}$ for $20 \mathrm{~s}$. The melting curve for each sample was carried out after 35 cycles. The relative expression patterns of SICBF1 were evaluated using the $2^{-\Delta \Delta C t}$ method [25].

\section{RESULTS and DISCUSSION}

\section{Physicochemical properties of CBF proteins}

The molecular weight (MW) and isoelectric point (pl) of CBF proteins in tomato, Arabidopsis, wheat, barley, rice and potato were predicted as shown in table 1 . The results revealed that the length of studied CBFs ranged from 205 (tomato-CBF3) to 257aa (potato-CBF2). The pl value of CBFs in dicots plants (tomato, Arabidopsis and potato) was less than monocots plants (wheat, barley and rice) as the rice-CBF showed the highest value (10.18) and potato-CBF2 had the lowest value (4.54). The prediction of CBFs-subcellular location exhibited that the cellular location of Arabidopsis-CBF1-3 was in nucleus while the subcellular location of tomato-CBF1 was predicted in cytoplasm and nucleus.

\section{Phylogenetic analysis of CBFs}

To understand the phylogenetic relationship between CBFs in monocots and dicots plants, the phylogenetic tree was constructed based on multiple alignments of amino acid sequences of CBFs in tomato, Arabidopsis, wheat, barley, rice and potato (Fig. 1). According to the evolution analysis, the CBFs of dicots plants were visibility separated from monocots. The CBF proteins from tomato showed closer relationships to CBF3 protein from potato. Also, CBF1 and CBF2 in tomato exhibited close similarity as well as CBF1 and CBF2 proteins from Arabidopsis. 
Table 1- Properties of CBF proteins in Arabidopsis, tomato, potato, wheat, barley and rice

\begin{tabular}{ccccccc}
\hline $\begin{array}{c}\text { Gene accession } \\
\text { number }\end{array}$ & $\begin{array}{c}\text { Gene } \\
\text { name }\end{array}$ & Organism & $\begin{array}{c}\text { Length } \\
(\mathbf{a a})\end{array}$ & $\begin{array}{c}\text { MW } \\
\text { (KDa) }\end{array}$ & pl & $\begin{array}{c}\text { Predicted } \\
\text { location(s) }\end{array}$ \\
\hline AAS77820 & CBF1 & Solanum lycopersicum & 210 & 23.40 & 5.23 & Cyto*. Nucleus. \\
AAS77821 & CBF2 & Solanum lycopersicum & 220 & 24.60 & 5.33 & Nucleus. \\
AAS77819 & CBF3 & Solanum lycopersicum & 205 & 23.06 & 5.48 & Cyto. Nucleus. \\
AAV80413 & CBF1 & Arabidopsis thaliana & 213 & 23.82 & 5.08 & Nucleus \\
AAV80415 & CBF2 & Arabidopsis thaliana & 216 & 24.27 & 5.00 & Nucleus \\
AAV80414 & CBF3 & Arabidopsis thaliana & 216 & 24.25 & 5.08 & Nucleus \\
NP_001274856 & CBF2 & Solanum tuberosum & 257 & 28.45 & 4.54 & Cyto. Nucleus \\
AAL37944 & CBF & Triticum aestivum & 212 & 23.34 & 7.78 & Cyto. Nucleus \\
AKE47413 & CBF2 & Triticum aestivum & 225 & 25.14 & 5.11 & Cyto. Nucleus \\
AAG59618 & CBF & Hordeum vulgare & 249 & 26.33 & 5.33 & Cyto. Nucleus \\
AAG59619 & CBF & Oryza sativa & 253 & 27.67 & 10.18 & Cyto. \\
\hline
\end{tabular}

Cyto: cytoplasm

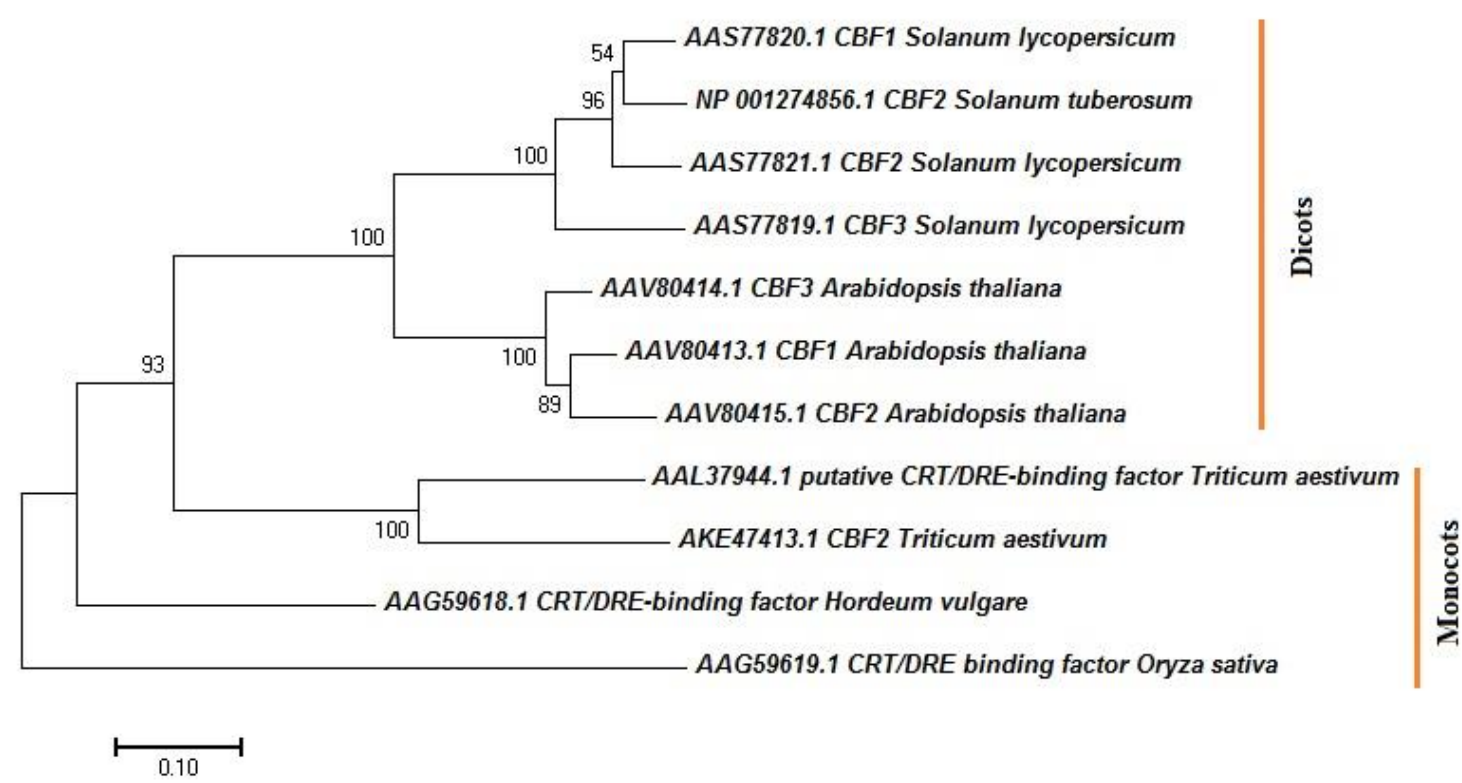

Figure 1- Phylogenetic analysis of CBF proteins using neighbor-joining method

\section{Protein-protein interactions in CBFs}

The interaction network of CBF genes in tomato and Arabidopsis revealed that Solyc03g082820 (uncharacterized protein) just had an interaction with CBF1-3 from tomato while Arabidopsis-CBF1-3 exhibited a high interaction with each other (Fig. 2). Arabidopsis is model plant that its molecular aspects have been extensively investigated more than tomato. CBF1 from tomato had an interaction with THM18 (also known as SIMYB14) which is a member of R2R3MYB gene family that involves in plant response to environmental stresses. The some key cold-response proteins such as ICE1, COR15A and HOS1 exhibited high interaction with Arabidopsis-CBF1-3. ICE1 is an inducer of CBF genes in Arabidopsis that it binds to MYB elements of CBFs promoter under cold stress [26]. ICE1 is upstream of cold responsive pathway and regulates the key cold regulated genes such COR15A and CBF genes [26, 27]. HOS1 was observed as a member of CBFs interaction 
network that interacts with ICE1. HOS1 is a negative regulator of cold responses and it involve in ubiquitination and degradation of ICE1 as an E3 ligase [28].
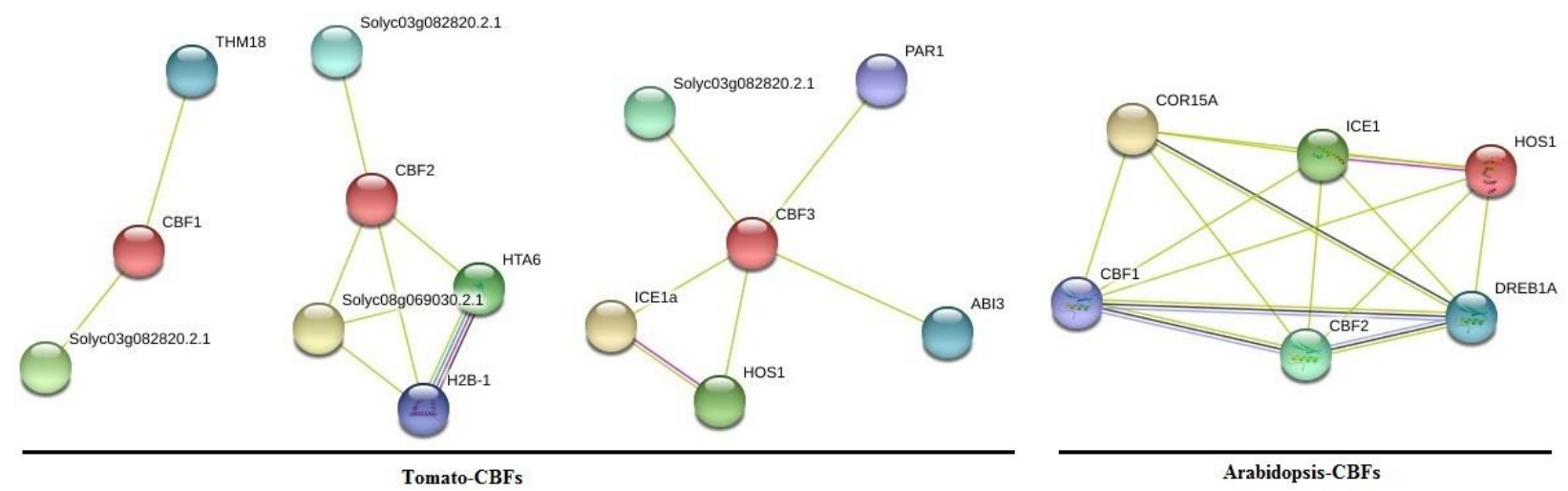

Figure 2- Proteins interaction network of CBF proteins in tomato and Arabidopsis

\section{Promoter analysis}

Cis-regulatory elements, such as promoters and enhancers, control physiology and development by regulating gene expression [2, 29]. The different cis-regulatory elements were found in upstream sequences (1000bp) of SICBF1and AtCBF1 that they involve in biotic and abiotic stresses (Fig. 3). The GARE motif (gibberellin-responsive element), CGTCA-motif (cis-acting regulatory element involved in the MeJA-responsiveness) and TC-rich repeats (cis-acting element involved in defense and stress responsiveness) were distributed within the regulatory region of SICBF1 and AtCBF1 genes. Some key regulatory elements such as HSE (cis-acting element involved in heat stress responsiveness) and MYS (MYB binding site involved in drought-inducibility) were observed in upstream of SICBF1. The ABRE and AuxRR which involve in abscisic acid (ABA) and auxin hormones signaling respectively were found in promoter region of AtCBF1. ABRE is an ABA response element that plays critical role in abiotic stresses [30]. However CBF1 is expressed by ABA independent pathway [26]. The identification of cis-regulatory DNA elements responsive to stress is important to determine gene regulatory mechanisms under various stresses [2, 31]. The present result revealed that SICBF1 and AtCBF1 have different regulatory elements in their promoter region which caused different-induction patterns.

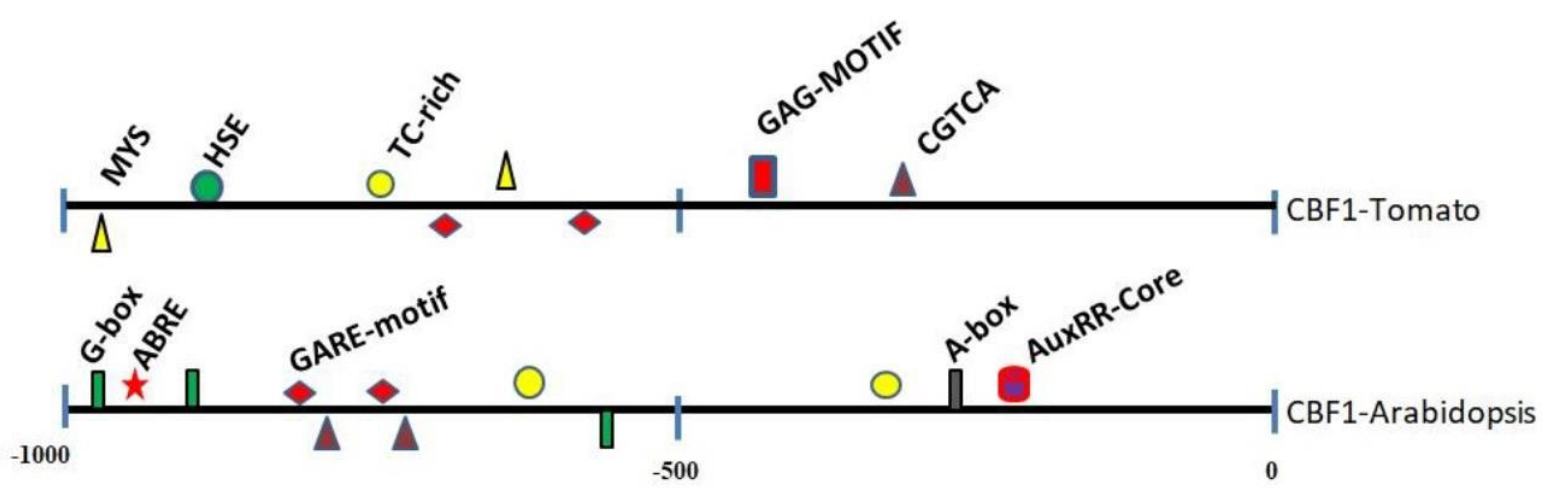

Figure 3- Distribution of cis-acting regulatory elements in the $5^{\prime}$ regulatory sequences of CBF1in tomato and Arabidopsis 


\section{Expression patterns of SICBF1 gene in tomato genotypes}

To more understand the role of SICBF1 under cold stress, the expression patterns of SICBF1 gene were evaluated at 10 and $4^{\circ} \mathrm{C}$ in sensitive and tolerant tomato genotypes (Fig. 4). The SICBF1 gene exhibited the different expression patterns in tomato genotypes whereas SICBF1 was down regulated in Moneymaker (as cold sensitive) at 10 and $4^{\circ} \mathrm{C}$ after 3 days but it was up-regulated at $4^{\circ} \mathrm{C}$ in LA1777 (as cold tolerant genotypes). CBF1 is key transcription factor that involve in plant response to low temperature, and drought stress [3]. Zhang et al. and Liu et al. also reported that SICBF1 was induced under cold stress $\left(4^{\circ} \mathrm{C}\right)[15$, 32]. It is not surprising to see that SICBF1 gene was not induced at $10{ }^{\circ} \mathrm{C}$ in cold tolerant genotype that probably $S I C B F 1$ is not induced in low temperature when it decreased to $4{ }^{\circ} \mathrm{C}$, SICBF1 was stimulated. To compare the SICBF1 with Arabidopsis-CBF1-3, the expression profiles of AtCBF1, AtCBF2 and AtCBF3 were evaluated in cold, drought and salt stresses using Arabidopsis microarray data. AtCBF1, AtCBF2 and AtCBF3 were highly expressed under cold stress (Fig. 5). AtCBF1 gene was found to be more expressed in cold stress than AtCBF2 and AtCBF3 while it was less induced in drought and salt stresses than others. In Arabidopsis, CBF1 is an early response and induces the CBF2 and CBF3 that involve stimulating the cold responsive genes [33]. It seems that AtCBF1 is main transcriptional activator during cold temperature and overexpressed AtCBF1 could increase the cold tolerance [34]. According to results of gene expression patterns, AtCBF1 is more involved to induce the key cold-regulated genes than SICBF1. It seems that the regulatory mechanisms of tomato are probably different from Arabidopsis

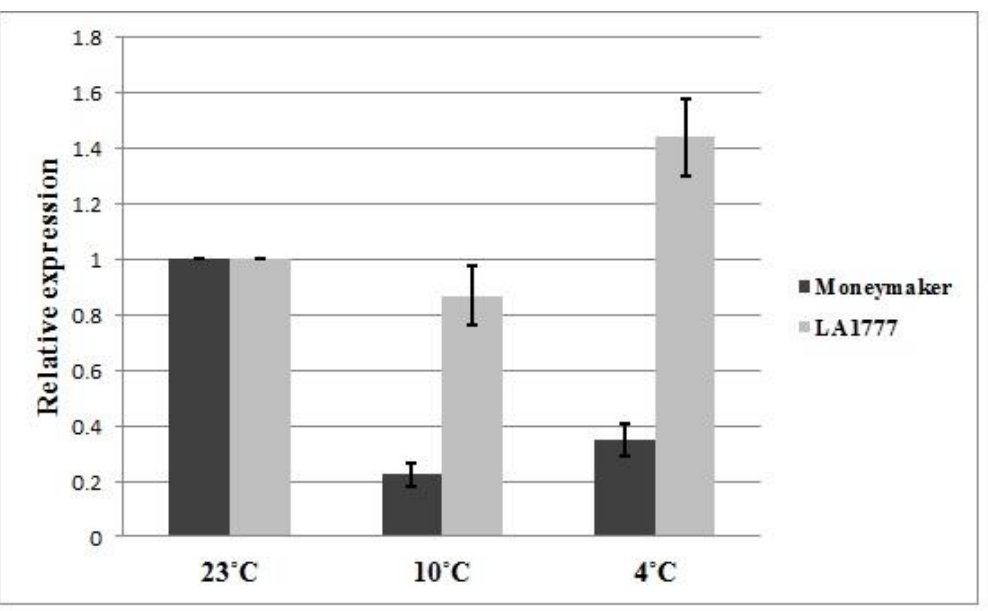

Figure 4- The SICBF1 expression patterns under cold stresses (10 and $4^{\circ} \mathrm{C}$ ) after 3 days in Moneymaker (as cold sensitive genotype) and LA1777 (as cold tolerant genotype). 


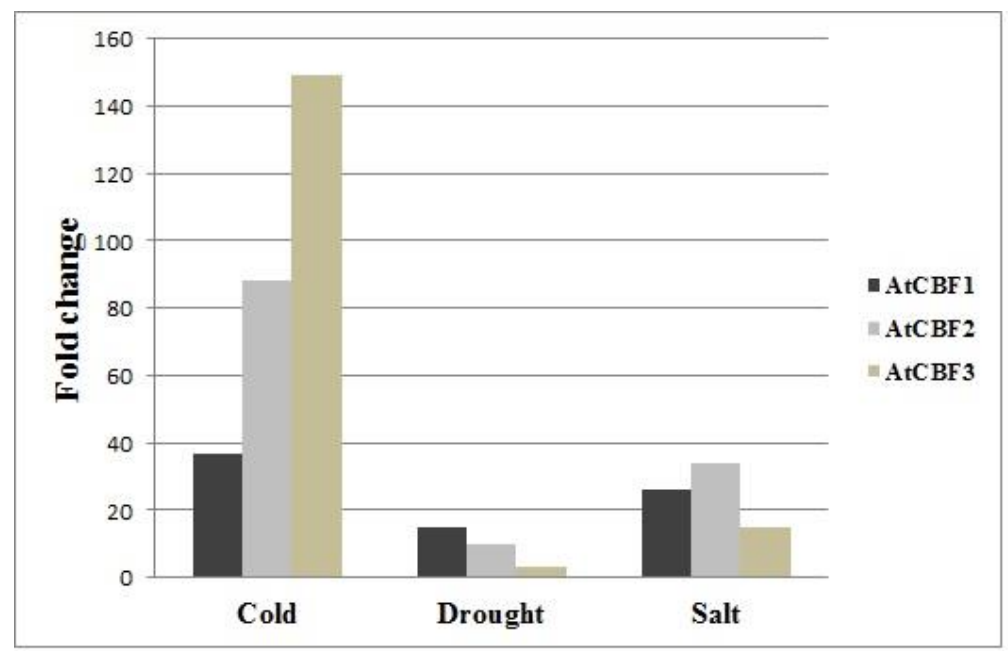

Figure 5- The expression profile of Arabidopsis-CBF1-3 under cold, drought and salt stresses based on microarray data using Genevestigator database

\section{CONCLUSION}

In this study, CBF1-3 from Arabidopsis and tomato were compared based on properties of protein sequence, phylogenetic analysis, interaction network and types of cis regulatory elements in promoter region. The results showed that tomato-CBFs are similar to Arabidopsis-CBFs based on amino acid sequences but they have different cis regulatory elements in promoter sites which could effect on their regulatory mechanisms. To better understand the function of CBF genes, the expression patterns of SICBF1 were considered at10 and $4^{\circ} \mathrm{C}$ in two contrasting tomato genotypes using qPCR that SICBF1 exhibited differential expression in tomato genotypes. Present study revealed that CBF genes from Arabidopsis are more involved in cold stress than tomato-CBFs. The different factors such as promoter sequences and protein-protein interactions could affect on CBF functions.

Acknowledgments: The author appreciates the Shahrood University of Technology for providing financial and facilities to carry out this research work.

Conflicts of Interest: The author declares that i have no conflict of interest.

\section{REFERENCES}

1. Nakashima K, Ito Y, Yamaguchi-Shinozaki K. Transcriptional regulatory networks in response to abiotic stresses in Arabidopsis and grasses. Plant Physiol. 2009; 149: 88-95.

2. Ahmadizadeh $M$, Heidari $P$. Bioinformatics study of transcription factors involved in cold stress. Biharean Biologist. 2014; 8 (2): 83-86.

3. Li Z, Li J, Xu X, Yao Q, Wang A. Isolation and functional characterization of the ShCBF1 gene encoding a CRT/DRE-binding factor from the wild tomato species Solanum habrochaites. Plant Physiol Bioch. 2014; 74: 294-303.

4. Izadi F, Nikfekr R, Soorni J. Transcription Factors-microRNAs regulatory network in response to multiple stresses in Arabidopsis thaliana. POJ. 2017; 10(04):183-189.

5. Licausi F, Ohme-Takagi M, Perata P. APETALA2/Ethylene Responsive Factor (AP2/ERF) transcription factors: mediators of stress responses and developmental program. New Phytol. 2013; 199(3): 639-649.

6. Thomashow MF. Molecular basis of plant cold acclimation: Insights gained from studying the CBF cold response pathway. Plant Physiol. 2010; 154: 571-577. 
Heidari, P.

7. Gilmour SJ, Fowler SG, Thomashow MF. Arabidopsis transcriptional activators CBF1, CBF2, and CBF3 have matching functional activities. Plant Mol Biol. 2004; 54: 767-781.

8. Yamaguchi-Shinozaki K, Shinizaki K. A novel cis-acting element in an Arabidopsis gene is involved in responsiveness to drought, low-temperature or high-salt stress. Plant Cell. 1994; 6: 251-164.

9. Xu ZS, Ni ZY, Li ZY, Li LC, Chen M, Gao D Y, et al. Isolation and functional characterization of HvDREB1-a gene encoding a dehydration-responsive element binding protein in Hordeum vulgare. J. Plant Res. 2009; 122: 121-130.

10. Dubouzet JG, Sakuma Y, Ito Y, Kasuga M, Dubouzet EG, Miura S, et al. OsDREB genes in rice, Oryza sativa L., encode transcription activators that function in drought-, high-salt- and cold-responsive gene expression. Plant J. 2003; 33: 751-763.

11. Novillo F, Alonso JM, Ecker JR, Salinas J. CBF2-DERB1C is a negative regulator of cbf1_DERB1B and CBF3_DERB1A expression and plays a central role in stress tolerance in Arabidopsis. PNAS. 2004; 101(11): 3985-90.

12. Liu Q, Kasuga M, Sakuma $Y$, Abe H, Miura S, Yamaguchi-Shinozaki K, et al. Two transcription factors, DREB1 and DREB2, with an EREBP/AP2 DNA binding domain separate two cellular signal transduction pathways in drought- and low-temperature-responsive gene expression, respectively, in Arabidopsis. Plant Cell. 1998; 10(8): 1391-406.

13. Shen YG, Zhang WK, He SJ, Zhang JS, Liu Q, Chen SY. An EREBP/AP2-type protein in Triticum aestivum was a DRE-binding transcription factor induced by cold, dehydration and ABA stress. Theor Appl Genet. 2003; 106: 923-930.

14. Caffagni A, Pecchioni N, Francia E, Pagani D, Milc J. Candidate gene expression profiling in two contrasting tomato cultivars under chilling stress. Biol Plantarum. 2014; 58: 283-295.

15. Zhang X, Fowler SG, Cheng HM, Lou YG, Rhee SY, Stockinger EJ, et al. Freezing-sensitive tomato has a functional CBF cold response pathway, but a CBF regulon that differs from that of freezing-tolerant Arabidopsis. Plant J. 2004; 39: 905-919.

16. Li R, Zhang L, Wang L, Chen L, Zhao R, Sheng J, Shen L. Reduction of Tomato-Plant Chilling Tolerance by CRISPR-Cas9-Mediated SICBF1 Mutagenesis. J Agr Food Chem. 2018; 66 (34): 9042-9051.

17. Benson D A, Cavanaugh M, Clark K, Karsch-Mizrachi I, Lipman D J, Ostell J, et al. GenBank. Nucleic Acids Res. 2013; 41(D1): D36-D42.

18. Gasteiger E, Hoogland C, Gattiker A, Duvaud S, Wilkins MR, Appel RD, et al. Protein Identification and Analysis Tools on the ExPASy Server. The Proteomics Protocols Handbook, Humana Press: 2005; 571-607.

19. Chou KC, Shen HB. Plant-mPLoc: a top-down strategy to augment the power for predicting plant protein subcellular localization. PLOS ONE. 2010; 5: e11335.

20. Szklarczyk D, Morris JH, Cook H, Kuhn M, Wyder S, Simonovic M, et al. The STRING database in 2017: quality-controlled protein-protein association networks, made broadly accessible. Nucleic Acids Res. 2017; 45: D362-68.

21. Kumar S, Stecher G, Tamura K. MEGA7: Molecular Evolutionary Genetics Analysis version 7.0 for bigger datasets. Mol Biol Evol. 2016; 33(7): 1870-1874. 
22. Lescot M1, Déhais P, Thijs G, Marchal K, Moreau Y, Van de Peer $Y$, et al. PlantCARE, a database of plant cis-acting regulatory elements and a portal to tools for in silico analysis of promoter sequences. Nucleic Acids Res. 2002; 30(1): 325-327.

23. Hruz T, Laule O, Szabo G, Wessendorp F, Bleuler S, Oertle L, et al. Genevestigator V3: a reference expression database for the meta-analysis of transcriptomes. Advances in Bioinformatics. 2008; ID 420747.

24. Untergasser A, Nijveen $H$, Rao X, Bisseling T, Geurts R, Leunissen JA. Primer3Plus, an enhanced web interface to Primer3. Nucleic Acids Res. 2007; 35: W71-W74.

25. Livak KJ, Schmittgen TD. Analysis of Relative Gene Expression Data Using Real-Time Quantitative PCR and the $2-\triangle \Delta$ ct Method. METHODS. 2001; 25: 402-408.

26. Chinnusamy V, Zhu J, Zhu JK. Cold stress regulation of gene expression in plants. Trends Plant Sci. 2007; 12: 444-451.

27. Zarka DG, Vogel JT, Cook D, Thomashow MF. Cold induction of Arabidopsis CBF genes involves multiple ICE (inducer of CBF expression) promoter elements and a cold-regulatory circuit that is desensitized by low temperature. Plant Physiol. 2003; 133(2):910-8.

28. Dong $\mathrm{CH}$, Agarwal M, Zhang $\mathrm{Y}$, Xie Q, Zhu JK. The negative regulator of plant cold responses, HOS1, is a RING E3 ligase that mediates the ubiquitination and degradation of ICE1. PNAS. 2006; 103(21):8281-6.

29. Wittkopp PJ, Kalay G. Cis-regulatory elements: molecular mechanisms and evolutionary processes underlying divergence. Nat Rev Genet. 2012; 13: 59-69.

30. Choi H, Hong J, Ha J, Kang J, Kim SY. ABFs, a family of ABA-responsive element binding factors. J. Biol. Chem. 2000; M275: 1723-1730.

31. Agarwal PK, Jha B. Transcription factors in plants and ABA dependent and independent abiotic stress signaling. Biol Plantarum. 2010; 54: 201-212.

32. Liu H, Ouyang B, Zhang J, Wang T, Li H, Yuyang Z, et al. Differential Modulation of Photosynthesis, Signaling, and Transcriptional Regulation between Tolerant and Sensitive Tomato Genotypes under Cold Stress. PLOS ONE. 2012; 7(11): e50785.

33. Gilmour SJ, Sebolt AM, Salazar MP, Everard JD, Thomashow MF. Overexpression of the Arabidopsis CBF3 transcriptional activator mimics multiple biochemical changes associated with cold acclimation. Plant Physiol. 2000; 124: 1854-1865.

34. Jaglo-Ottosen KR, Gilmour SJ, Zarka DG, Schabenberger O, Thomashow MF. Arabidopsis CBF1 overexpression induces COR genes and enhances freezing tolerance. Science. 1998; 280: 104-106.

( 2018 by the authors. Submitted for possible open access publication under the terms and conditions of the Creative Commons Attribution (CC BY NC) license (https://creativecommons.org/licenses/by-nc/4.0/). 